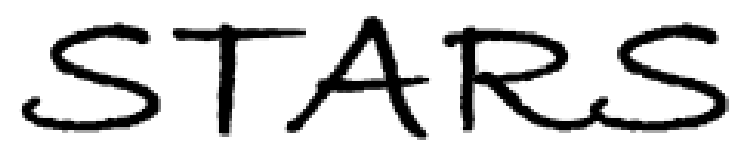

University of Central Florida

STARS

$1-1-2010$

\title{
Multiplexed biomarker detection using x-ray fluorescence of composition-encoded nanoparticles
}

\author{
Mainul Hossain \\ University of Central Florida \\ Chaoming Wang \\ University of Central Florida \\ Ming Su \\ University of Central Florida
}

Find similar works at: https://stars.library.ucf.edu/facultybib2010 University of Central Florida Libraries http://library.ucf.edu

This Article is brought to you for free and open access by the Faculty Bibliography at STARS. It has been accepted for inclusion in Faculty Bibliography 2010 s by an authorized administrator of STARS. For more information, please contact STARS@ucf.edu.

\section{Recommended Citation}

Hossain, Mainul; Wang, Chaoming; and Su, Ming, "Multiplexed biomarker detection using x-ray fluorescence of composition-encoded nanoparticles" (2010). Faculty Bibliography 2010s. 267. https://stars.library.ucf.edu/facultybib2010/267

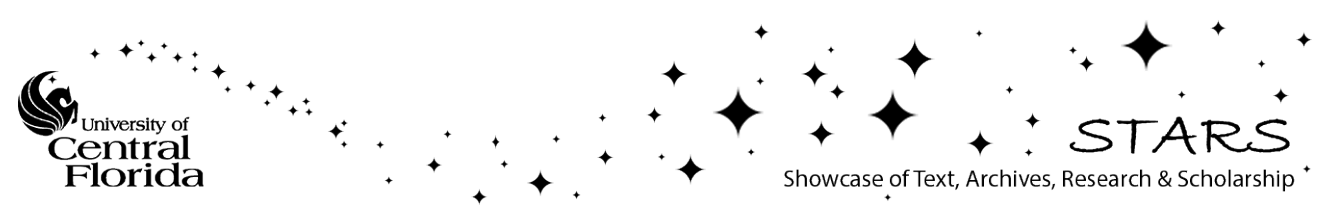




\section{Multiplexed biomarker detection using x- ray fluorescence of composition-encoded nanoparticles}

Cite as: Appl. Phys. Lett. 97, 263704 (2010); https://doi.org/10.1063/1.3533817

Submitted: 30 November 2010 . Accepted: 13 December 2010 . Published Online: 30 December 2010

Mainul Hossain, Chaoming Wang, and Ming Su

\section{ARTICLES YOU MAY BE INTERESTED IN}

Multiplexed highly sensitive detections of cancer biomarkers in thermal space using encapsulated phase change nanoparticles

Applied Physics Letters 95, 043701 (2009); https://doi.org/10.1063/1.3189082

NanoSIMS for biological applications: Current practices and analyses

Biointerphases 13, 03B301 (2018); https://doi.org/10.1116/1.4993628

\section{Applied Physics Reviews} Now accepting original research 


\title{
Multiplexed biomarker detection using x-ray fluorescence of composition- encoded nanoparticles
}

\author{
Mainul Hossain, ${ }^{1,2}$ Chaoming Wang, ${ }^{1,2}$ and Ming Su ${ }^{1,2, a)}$ \\ ${ }^{1}$ NanoScience Technology Center, University of Central Florida, Orlando, Florida 32826, USA \\ ${ }^{2}$ School of Electrical Engineering and Computer Science, University of Central Florida, Orlando, \\ Florida 32826, USA
}

(Received 30 November 2010; accepted 13 December 2010; published online 30 December 2010)

\begin{abstract}
Multiple DNA and protein biomarkers have been detected based on characteristic x-ray fluorescence of a panel of metal and alloy nanoparticles, which are modified with ligands of biomarkers to create a one-to-one correspondence and immobilized on ligand-modified substrates after forming complexes with target biomarkers in three-strand or sandwich configuration. By determining the presence and concentration of nanoparticles using x-ray fluorescence, the nature and amount of biomarkers can be detected with limits of $1 \mathrm{nM}$ for DNA and $1 \mathrm{ng} / \mathrm{ml}$ for protein. By combining high penetrating ability of $\mathrm{x}$-rays, this method allows quantitative imaging of multiple biomarkers. (C) 2010 American Institute of Physics. [doi:10.1063/1.3533817]
\end{abstract}

Detecting DNA and protein biomarkers with high sensitivity allows early detection of cancers. Although many cancer biomarkers with certain specificity have been found, it is clear that no single biomarker is likely to distinguish lethal cancer from indolent ones at early stage due to lack of tumor specificity. ${ }^{1}$ Parallel to efforts to find specific biomarkers, a feasible way of enhancing reliability is to detect multiple biomarkers with high sensitivity at the same time. ${ }^{2-4}$ The multiplexed detection reflects functional relation among biomarkers, and identifies biomarkers associated with different stages of disease, allowing early detection of lethal cancers. Nanoparticles with unique optical, electrical, magnetic, or electrochemical signatures can be used for biomarker detection owing to their intimate contact with biomolecules. ${ }^{5}$ Extremely high sensitivities have been achieved at pmol/l or fmol/l levels by converting biorecognition events into measurable physical signals that can be amplified. But, the multiplicity of nanoparticle detections is limited due to narrow operating range and broad peak: only one or few types of biomarkers can be detected at one time; and screening multiple biomarkers in a sample will take a long time, and a lot of agents and effort. ${ }^{6}$ The interaction of incoming x-rays with atoms generates characteristic $\mathrm{x}$-ray fluorescence (XRF), which can be used to quantify trace elements in tissues such as bones and cancers at high sensitivity and specificity. ${ }^{7-15}$ Though XRF has shown great potential for tissue imaging, it has not been applied to biomarker detection, and tissue imaging by intentionally introducing nanoparticles.

This paper describes an XRF based technique for multiplexed biomarker detection using a panel of metal and alloy nanoparticles. These nanoparticles are modified with ligands to establish a one-to-one correspondence, and immobilized on a substrate that has been modified with ligands through ligand-biomarker interaction (Fig. 1). The nature and amount of multiple biomarkers can be detected at high sensitivity by determining the presence and amount of according nanoparticles with characteristic XRF. The high penetrating power and nondestructive nature of $\mathrm{x}$-rays also allow quantitative imaging of multiple biomarkers, where results can be ob-

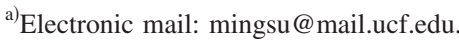

tained instantaneously with high spectral resolution. The nanoparticles are very stable under physiological conditions even without silica or polymeric coating, and can be used for either in vitro detection or in vivo imaging, or biomarker profiling in tissue samples. ${ }^{16}$

Metallic nanoparticles of metals and alloys such as indium, bismuth, tin, and lead-tin alloy nanoparticles are made by thermal decompositions of organometallic precursors in ethylene glycol in the presence of a surfactant (polyvinylpyrrolidone) at $200{ }^{\circ} \mathrm{C}$ under nitrogen protection. The nanoparticles are surface-oxidized by heating at $110{ }^{\circ} \mathrm{C}$ for 10 min in ambient condition, and aminized by incubating in 5\% alcoholic solution of (aminopropyl)triethoxy-silane for $1 \mathrm{~h}$. After removing excess silane by centrifugation, aminized nanoparticles are suspended in dimethyl sulfoxide (DMSO) containing 5\% N-succinimidyl (4-iodoacetyl) aminobenzoate (SIAB) for $20 \mathrm{~min}$. After removing excess SIAB, nanoparticles are incubated with thiolated probe single strand DNA (ssDNA) in phosphate buffer solution (PBS) $(p \mathrm{H} \mathrm{7.0)}$ at room temperature for $3 \mathrm{~h}$. Amine-modified nanoparticles are then incubated with disuccinimidyl suberate (DSS) in DMSO for $1 \mathrm{~h}$. After removing excess DSS, nanoparticles are incubated with antibody inside PBS for $3 \mathrm{~h}$. Solid substrates (i.e., aluminum plate) are modified with capture ssDNA or antibody using similar methods described above.

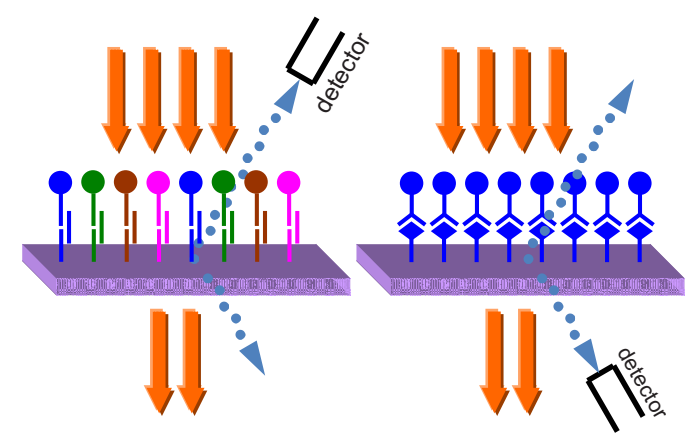

FIG. 1. (Color online) Biomarker detection based on XRF of compositionencoded nanoparticles, where three-strand detection of oligonucleotides and sandwich detection of protein are carried out in reflection mode and transmission mode, respectively. 

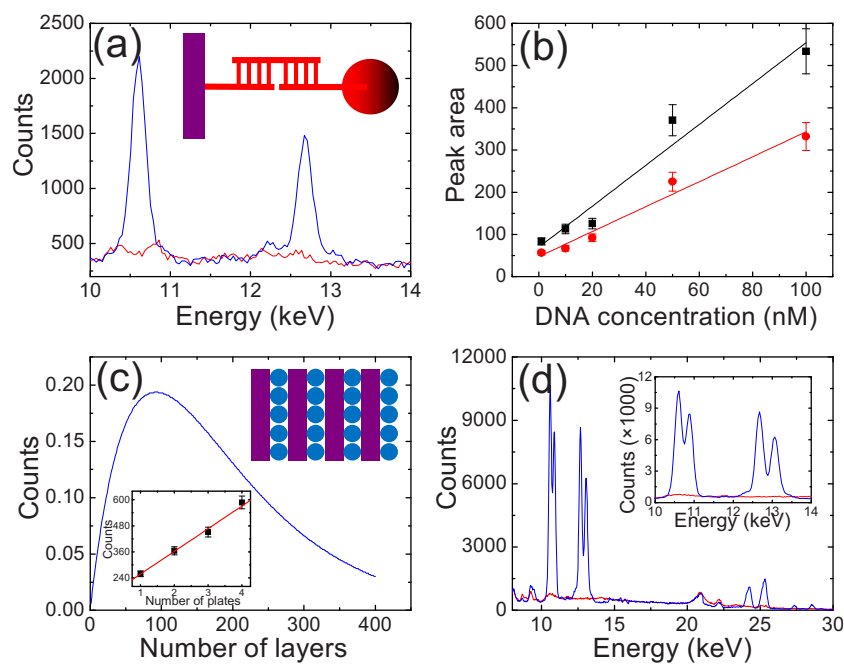

FIG. 2. (Color online) (a) XRF spectrum of lead-tin alloy nanoparticles after detecting $100 \mathrm{nM}$ ssDNA, where the bottom and top lines are background and signal, respectively; (b) the peak areas of lead $L_{\alpha 1}$ (top) and $L_{\beta 1}$ (bottom) as a function of ssDNA concentration; (c) the intensity of bismuth $L_{\alpha 1}$ peak as a function of ssDNA concentrations, where four aluminum plates are stacked together to enhance signal intensity in transmission mode (inset); (d) XRF spectrum of lead (10.55, $12.61 \mathrm{keV})$, bismuth $(10.84,13.02$ $\mathrm{keV})$, indium $(24.21,27.27 \mathrm{keV})$, and tin $(25.27,28.48 \mathrm{keV})$ nanoparticles after detecting four types of ssDNAs at concentrations of $100 \mathrm{nM}$, where (d) inset shows lead and bismuth $L$ peaks at higher magnification, where bottom and top lines are background and signal, respectively.

A Mini-X x-ray tube (Amptek, Bedford, MA) operating at $40 \mathrm{kV}$ and $15 \mu \mathrm{A}$ is used to generate primary $\mathrm{x}$-rays. The tube is fitted with a brass collimator to reduce beam size to 2 $\mathrm{mm}$ in diameter. An x-ray spectrometer (Amptek X-123) with Si-PIN photodiode is used to analyze XRF emissions. The spectrometer contains a detector, a digital pulse processor, and a multichannel analyzer, interfaced with a computer for data acquisition and analysis. XRF from nanoparticles is detected in both transmission and reflection modes (Fig. 1). In transmission mode, the x-ray tube and the detector are aligned in the same line; while in reflection mode, the tube and the detector are fixed on a mounting plate with an angle of $45^{\circ}$ between them. The tube-sample distance and sampledetector distance are kept at 3 and $2 \mathrm{~cm}$, respectively. The whole setup is enclosed in a lead containing acrylic chamber with $1 \mathrm{~mm}$ of lead equivalent thickness. The primary $40 \mathrm{kV}$ $\mathrm{x}$-rays can excite electrons with binding energy below 40 $\mathrm{keV}$; thus only $L$ line emissions can be excited in case of high atomic number nanoparticles (i.e., lead and bismuth). Background spectra are obtained using an aluminum plate without nanoparticles.

The detection is carried out in three-strand configuration (DNA) or sandwich configuration (protein). Ligand-modified aluminum plates are immersed in PBS solutions containing target ssDNA at $p \mathrm{H} 7.0$ for $3 \mathrm{~h}$. The plates are washed with PBS $(p \mathrm{H}$ 7.0) for three times, dried in air, and tested using the XRF setup. Figure 2(a) shows lead $L_{\alpha 1}$ line $(10.55 \mathrm{keV})$ and lead $L_{\beta 1}$ line $(12.61 \mathrm{keV})$ from lead-tin nanoparticles (60\% lead and $40 \%$ tin by mass) immobilized on an aluminum plate after detecting $100 \mathrm{nM}$ DNA, where the two $L$ peaks have different intensities due to difference in fluorescence yields. The intensity ratio of $L_{\beta 1} / L_{\alpha 1}$ lines is determined to be $\sim 0.7$, which is close to the ratio of their fluorescence yields $(\sim 1.0) .{ }^{17}$ Figure 2 (b) shows dependences of peak area on DNA concentration for both peaks in the range
1-100 nM, which is obtained after removing background, and integrating area under each peak. The top line represents the area under the $L_{\alpha 1}$ peak and the bottom line represents the area under the $L_{\beta 1}$ peak. The slope of each line is a measure of the detection sensitivity for the according $L$ line, and is found to be higher for the larger $L_{\alpha 1}$ peak compared to the smaller $L_{\beta 1}$ peak. A similar method has been used to detect ssDNA using indium, tin, or bismuth nanoparticles (not shown). Providing other conditions (nanoparticle diameter, collection time, x-ray flux, and detector-sample distance) are identical, lead-tin alloy and bismuth nanoparticles provide the highest detection sensitivities compared to other nanoparticles due to their higher fluorescence yield.

The sensitivity of detection can be enhanced by stacking substrates with nanoparticles, and detecting x-ray emission in transmission mode. Several aluminum plate with immobilized bismuth nanoparticles after detecting DNA are stacked, and Fig. 2(c) inset shows the intensity of the $L_{\alpha 1}$ peak as a function of number of stacked plates. A linear relation exists when the total number of layers is smaller than four. The limit on the number of stacking plates is derived as follows: (1) x-ray absorbance by aluminum and bismuth nanoparticles is based on Lambert-Beer law, and (2) generation of x-ray fluoroscence is proportional to thickness (layers) of bismuth nanoparticles on aluminum plates. Provided that primary $\mathrm{x}$-ray is monochromic and has energy of $40 \mathrm{keV}$, the net intensity $(I)$ of fluorescence emission can be derived using

$$
I=\omega n\left(d_{1}+d_{2}\right) \exp \left[-n\left(\mu_{1} d_{1}+\mu_{2} d_{2}\right)\right],
$$

where $\omega$ is the fluorescence yield of bismuth $L_{\alpha 1}(0.37), n$ is the number of stacking nanoparticle-aluminum layers, and $d$ and $\mu$ are the thickness (or diameter) and absorption coefficients of aluminum (1) and bismuth (2), respectively. The solid line in Fig. 2(c) shows simulated result, where the thickness of the aluminum plate is $0.15 \mathrm{~mm}$, the diameter of the bismuth nanoparticles is $10 \mathrm{~nm}$, and nanoparticles form a monolayer on the plate. After reaching the maximal layers (80), the fluorescence emission decreases, meaning that the maximum number of layers will be 80 , which corresponds to a sensitivity of $0.125 \mathrm{nM}$ for DNA detection.

Multiple ssDNAs are detected using bismuth, lead-tin, tin, and indium nanoparticles at the same time, where an aluminum plate is modified with a mixture of four capture ssDNAs at equal amounts. Figure 2(d) shows characteristic XRF peaks of four different nanoparticles after detecting four types of target ssDNAs, where lead $(10.55,12.61 \mathrm{keV})$, bismuth $(10.84,13.02 \mathrm{keV})$, indium $(24.21,27.27 \mathrm{keV})$, and tin $(25.27,28.48 \mathrm{keV})$ can be distinguished from background (red). The difference in fluorescence intensity is induced mainly by fluorescence yield, which is higher for elements with a higher atomic number. The Si-PIN detector that can resolve a $140-260 \mathrm{eV}$ peak at $5.9 \mathrm{keV}$ enables detection of lead $L_{\alpha 1}$ and $L_{\beta 1}(10.55$ and $12.61 \mathrm{keV})$ lines from bismuth $L_{\alpha 1}$ and $L_{\beta 1}$ lines $(10.84$ and $13.02 \mathrm{keV})$, as shown in the inset of Fig. 2(d). Based on energy resolution of detector $(200 \mathrm{eV})$, and energy range of detection $(0.5-30 \mathrm{keV})$, the number of possible peaks that can be detected will reach $\sim 150$, meaning that this method allows simultaneous detection of $\sim 150$ different peaks. On the other hand, the number of XRF peaks depends on how many different atoms are available in form of nanoparticles. From the Periodic Table, $\sim 50$ different types of elements could be made into nanopar- 

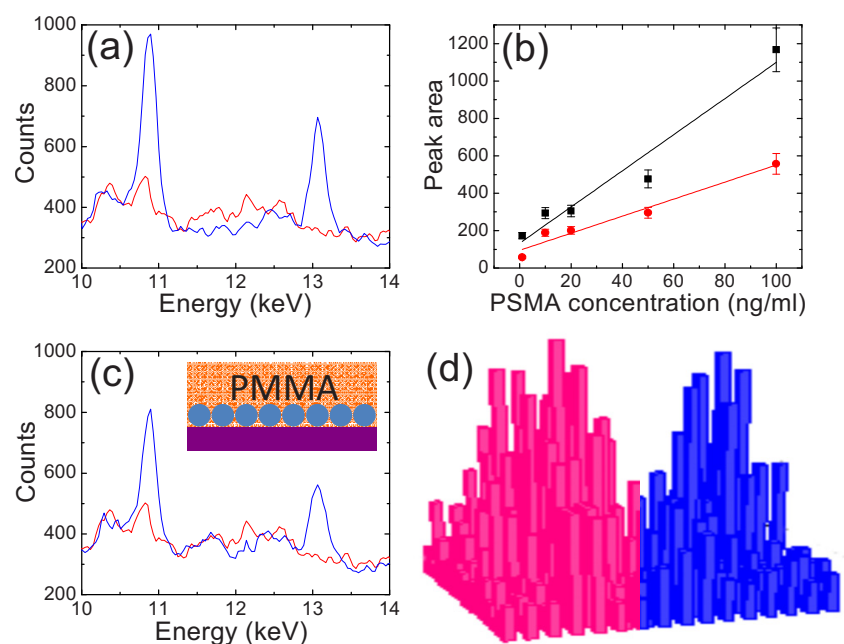

FIG. 3. (Color online) (a) XRF spectrum of bismuth nanoparticles after detecting $100 \mathrm{ng} / \mathrm{ml}$ of protein biomarker, where the bottom and top lines are background and signal, respectively; (b) the peak areas of bismuth $L_{\alpha 1}$ (top) and $L_{\beta 1}$ (bottom) as a function of protein concentration; (c) XRF spectrum of bismuth nanoparticles immobilized on an aluminum plate with $100 \mathrm{ng} / \mathrm{ml}$ of protein biomarker and then covered with $10 \mathrm{~mm}$ PMMA; (d) spatially distributed intensities of two discrete areas on a plastic substrate covered with bismuth and lead-tin nanoparticles.

ticles with characteristic $\mathrm{X}$-ray signatures; thus, the multiplicity of nanoparticle enabled XRF technique could reach $\sim 50$.

The feasibility of in vivo protein biomarker detection/ imaging using XRF has been studied after immobilizing prostate specific membrane antigens (PSMAs) onto substrates. Ligand-modified aluminum plates are immersed in PBS solutions containing antigen at $p \mathrm{H} 7.0$ for $3 \mathrm{~h}$; after

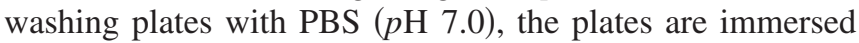
in PBS solution containing PSMA antibody modified bismuth nanoparticles for $3 \mathrm{~h}$. After removing excess nanoparticles by washing, the plates with immobilized nanoparticles are analyzed by XRF. Figure 3(a) shows the emission lines of bismuth at $10.84\left(L_{\alpha 1}\right)$ and $13.02\left(L_{\beta 1}\right) \mathrm{keV}$ above background, where difference in peak area is induced by difference in fluorescence yield. The areas of both peaks depend linearly on antigen concentration [Fig. 3(b)], from which the detection limit for PSMA by using bismuth nanoparticles is 1 $\mathrm{ng} / \mathrm{ml}$. The aluminum plates with DNA-linked bismuth nanoparticles have been coated with $10 \mathrm{~mm}$ thick polymethyl methacrylate (PMMA) in order to mimic tissues surrounding tumors. Figure 3(c) shows XRF peaks of bismuth exposed to $40 \mathrm{kV}$ x-rays, where the signal intensity does not decrease after polymer coating. Furthermore, XRF is used to image multiple biomarkers after immobilizing bismuth nanoparticles and lead-tin nanoparticles on a plastic substrate. The patterned areas cover a $20 \times 20 \mathrm{~mm}^{2}$ area with $2 \mathrm{~mm}$ separation, and are coated with $10 \mathrm{~mm}$ thick PMMA. A twodimensional scan is carried out over an area of $100 \mathrm{~mm}^{2}$ at 1 $\mathrm{mm}$ step size, and the peak intensities for bismuth (10.84 $\mathrm{keV})$ and lead $(10.55 \mathrm{keV})$ are recorded at each position. Figure 3(d) shows the spatial distribution of XRF intensity, where column height represents fluorescence count after removing background. Two distinct regions are identified with significant increase in fluorescence intensity, reflecting that $\mathrm{x}$-ray beam hits on bismuth or lead-tin region. In contrast to computed tomography $(\mathrm{CT})$ where thickness contrast is con- sidered, the energy resolving XRF can differentiate nanoparticles for multiplexed imaging.

X-ray dose is estimated by using PCXMC, a PC-based Monte Carlo simulation program, in which X-ray photons are followed as they randomly interact with phantoms according to probability distribution of processes such as photoelectric absorption, coherent (Rayleigh), or incoherent (Compton) scattering. ${ }^{18}$ This produces a large number of independent photon histories from which the mean values of energy depositions can be estimated and used to calculate doses. A total of 20000 photon histories have been generated at a tube voltage of $40 \mathrm{kV}$. In case only beryllium window is used as a filter, the Monte Carlo simulation results in a surface dose of $3 \mathrm{~Gy}$, which is equivalent to a total body dose of $0.47 \mu \mathrm{Gy}$, and an effective dose of $0.72 \mu \mathrm{Gy}$ for an average adult $(70 \mathrm{~kg})$ and these are lower than the average radiation doses in regular medical procedures such as an abdominal x-ray (1.4 mGy) or an abdominal CT scan (8.0 mGy).

This method has the potential to detect multiple cancer biomarkers inside the human body. Nanoparticles with suitable surface modification can be taken into body, and conjugated onto tumors through antigen-antibody interaction. The penetrating power of incoming x-rays can be tuned by using beams of higher energy. The dose absorbed by the body can be further reduced by using monochromatic beams or beams with less background by appropriate filtration. By quantitatively imaging multiple surface biomarkers of cancer cells, the reliability of in vivo cancer detection can be hopefully enhanced without much harmful effect to human.

This work has been supported by King Biomedical Research Program, National Science Foundation and Department of Defense.

${ }^{1}$ R. Etzioni, D. F. Penson, J. M. Legler, D. Tommaso, R. Boer, P. H. Gann, and E. J. Feuer, J. Natl. Cancer Inst. 94, 981 (2002).

${ }^{2}$ F. Wei, P. Patel, W. Liao, K. Chaudhry, L. Zhang, M. Arellano-Garcia, S. Hu, D. Elashoff, H. Zhou, S. Shukla, F. Shah, C.-M. Ho, and D. T. Wong, Clin. Cancer Res. 15, 4446 (2009).

${ }^{3}$ M. Arellano-Garcia, S. Hu, J. Wang, B. Henson, H. Zhou, D. Chia, and D. T. Wong, Oral Dis. 14, 705 (2008).

${ }^{4}$ D. V. Makarov, S. Loeb, R. H. Getzenberg, and A. W. Partin, Annu. Rev. Med. 60, 139 (2009)

${ }^{5}$ N. L. Rosi and C. A. Mirkin, Chem. Rev. (Washington, D.C.) 105, 1547 (2005).

${ }^{6}$ L. Ma, Y. Hong, Z. Ma, C. Kaittanis, J. M. Perez, and M. Su, Appl. Phys. Lett. 95, 043701 (2009).

${ }^{7}$ T. Magalhães, M. L. Carvalho, A. Von Bohlen, and M. Becker, Spectrochim. Acta, Part B 65, 493 (2010).

${ }^{8}$ M. P. Silva, A. Tomal, C. A. Pérez, A. Ribeiro-Silva, and M. E. Poletti, X-Ray Spectrom. 38, 103 (2009).

${ }^{9}$ Y. Hayashi and F. Okuyama, Ger. Med. Sci. 8, 107 (2010).

${ }^{10}$ A. Banas, W. M. Kwiatek, K. Banas, M. Gajda, B. Pawlicki, and T. Cichocki, JBIC, J. Biol. Inorg. Chem. 15, 1147 (2010).

${ }^{11}$ K. Geraki, M. J. Farquharson, and D. A. Bradley, Phys. Med. Biol. 49, 99 (2004).

${ }^{12}$ U. Majewska, D. Banaś, J. Braziewicz, S. Góźdź, A. Kubala-Kukuś, and M. Kucharzewski, Phys. Med. Biol. 52, 3895 (2007).

${ }^{13}$ J. Börjesson and S. Mattsson, Powder Diffr. 22, 130 (2007).

${ }^{14}$ H. Nie, D. Chettle, L. Luo, and J. O'Meara, Nucl. Instrum. Methods Phys. Res. B 263, 225 (2007).

${ }^{15}$ A. C. Todd and D. R. Chettle, Environ. Health Perspect. 102, 172 (1994).

${ }^{16}$ P. J. Endres, K. W. MacRenaris, S. Vogt, M. J. Allen, and T. J. Meade, Mol. Imaging 5, 485 (2006).

${ }^{17}$ A. Karabulut and A. Gürol, Nucl. Instrum. Methods Phys. Res. B 244, 303 (2006).

${ }^{18}$ A. Servomaa and M. Tapiovaara, Radiat. Prot. Dosim. 80, 213 (1998). 